\title{
Ischemic Colitis in a Patient With Systemic Lupus Erythematosus: Case Report and Review of Literature
}

\author{
Belen Asheber Tesfaye ${ }^{\mathrm{a}, \mathrm{b}}$, Josia Narda Henry ${ }^{\mathrm{a}}$, Angesom Kibreab ${ }^{\mathrm{a}}$, Andrew Sanderson ${ }^{\mathrm{a}}$
}

\begin{abstract}
Ischemic colitis is an uncommon complication in patients with systemic lupus erythematosus (SLE). In previously reported cases of colitis caused by SLE, intestinal vasculitis is implicated as the causative process, but is rarely confirmed histologically. We described a case of a 57-year-old African American woman who presented with abdominal pain due to ischemic colitis with small vessel vasculitis which was proven by colonoscopy with biopsy. The clinical course of the patient was improved with conservative management and discontinuation of immunosuppressive agents.
\end{abstract}

Keywords: Colitis; SLE; Iscemia

\section{Introduction}

Ischemic colitis, an acute abdominal disease, is caused by various predisposing factors. Although patients with connective tissue disorders, such as systemic lupus erythematosus (SLE), are at risk for various forms of colonic ischemia due to impairment of small vessel circulation to the large bowel caused by widespread vasculitis, ischemic colitis is an uncommon gastrointestinal complication in patients with SLE $[1,2]$. We report a case of a 57-year-old African American female with ischemic colitis associated with intestinal vasculitis and presented with increased SLE activity.

\section{Case Report}

A 57-year-old female with history of SLE, fibromyalgia, seizure disorder, asthma and hypertension was admitted for ab-

\footnotetext{
Manuscript accepted for publication March 31, 2014

${ }^{\text {a}}$ Howard University Hospital, USA

${ }^{\mathrm{b}}$ Corresponding author: Belen Tesfaye, Howard University Hospital,

USA. Email: belenaye@yahoo.com
}

doi: http://dx.doi.org/10.14740/jmc1759w dominal pain of one-day duration and vomiting. She has had no previous episodes of abdominal pain. She had been constipated for one week prior to admission. She had no history of diarrhea or blood in stool. She was diagnosed with SLE in her 20s and was being managed with plaquenil and steroids. Her SLE was initially marked by butterfly-like facial rash as well as pain and swelling in all her joints. She reported good disease control on plaquenil for almost 10 years. Azathioprine was started three months prior to presentation as a steroid sparing medication. Prednisone was slowly weaned off.

On the day of admission, the vital signs were normal. Physical examination revealed mild tenderness of the left lower abdomen but no peritoneal signs or organomegaly. Initial laboratory test results were as follows: white blood cell count, $3.1 \times 10^{9} / \mathrm{L}$ (normal, $4.5 \times 10^{9} / \mathrm{L}-10.5 \times 10^{9} / \mathrm{L}$ ); hemoglobin, $7.9 \mathrm{~g} / \mathrm{dL}$; platelet count, $336 \times 10^{9} / \mathrm{L}$ (normal, $\left.150 \times 10^{9} / \mathrm{L}-350 \times 10^{9} / \mathrm{L}\right)$; blood urea nitrogen, $10 \mathrm{mg} / \mathrm{dL}$; creatinine, $0.5 \mathrm{mg} / \mathrm{dL}$. The results of electrolytes, LFTS and coagulation studies were normal except albumin level of 2.1 $\mathrm{mg} / \mathrm{dL}$. Immunologic test showed increased activity of SLE: ESR, $97 \mathrm{~mm} / \mathrm{h}$ (normal, 0 - $20 \mathrm{~mm} / \mathrm{h}$ ); CRP, $308 \mathrm{mg} / \mathrm{dL}$ (normal, 0 - $0.3 \mathrm{mg} / \mathrm{dL}$ ); antinuclear antibody (ANA), a titer of 1:640 (normal, < 1:50); C3, 69 mg/dL (normal, 79 - $152 \mathrm{mg} /$ $\mathrm{dL}$ ); C4, $19 \mathrm{mg} / \mathrm{dL}$ (normal, 16 - $38 \mathrm{mg} / \mathrm{dL}$ ), antiphospholipid antibody $<14 \mathrm{U} / \mathrm{mL}$ (normal, $<14 \mathrm{U} / \mathrm{mL}$ ); anti-double stranded DNA, 2 (normal, $<4 \mathrm{IU} / \mathrm{mL}$ ), antithrombin III, 75\% (normal, 85-128\%). Abdominal CT showed thickening of the cecum and a portion of ascending colon with associated fat stranding and edema. Even though CT angiography and magnetic resonance angiography (MRA) were recommended they were not carried out due to history of severe allergy to contrast. Colonoscopy demonstrated severe inflammation of the mucosa extending from cecum to mid portion of ascending colon, with contact bleeding and irregularly shaped ulceration (Fig. 1). Rectum, sigmoid, descending and transverse colon appeared relatively free from disease. Biopsy specimens from the cecum and ascending colon revealed inflammatory cell infiltration and hemorrhage in the mucosa, and small vessel wall thickening with lymphocyte infiltration, and vasculitis was considered. Figure 2 shows a crypt abscess. The crypt is dilated and is filled with neutophils and necrotic debris. 


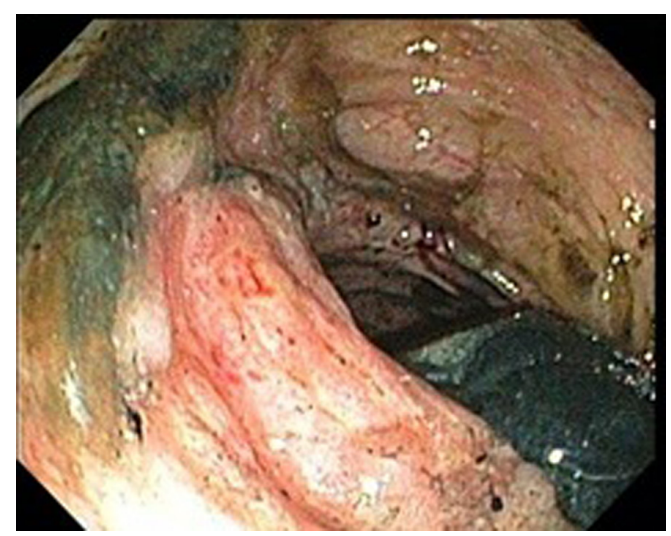

Figure 1. Severe inflammation and ulceration of the cecal wall.

Ischemic colitis with increased SLE activity was confirmed and conservative management was done with fluids and intravenous antibiotics. Azathioprine and plaquenil were discontinued due to progressive worsening in leucopenia per rheumatology recommendations. Clinical improvement was observed over the next week, with improvement of abdominal pain and leucopenia.

\section{Discussion}

The development of ischemic colitis in patients with SLE is an uncommon complication $[1,2]$. But widespread fibrinoid vasculitis, typical of SLE, is thought to be a likely predisposing factor [1]. If this vasculitis involves the colon, ischemic colitis occurs. Gastrointestinal vasculitis is one of the most serious complications of SLE, even though the occurrence of colonic lesions is rare $(0.2 \%)[3,4]$. The gastrointestinal vasculitis of SLE is consequence of tissue damage from vasculopathy mediated by immune complexes, and has been associated with SLE activity $[3,5]$. There are no pathognomic and histopathologic findings in SLE; however, pathologic changes associated with gastrointestinal vasculitis occur in the small vessels of the intestinal wall rather than in medium-sized mesenteric arteries [3, 4]. Ischemic colitis in patients with SLE is caused by decreased blood perfusion of mesenteric vasculatures. The predisposing factors are embolism, thrombosis, vasospasm, drugs (steroids and immunosuppressive agents), vasculitis, performed colonoscopy and enema $[1,2,6]$. Gomella et al studied the effects of immunosuppressive agents and ischemic colitis on rats. One treatment group received solumedrol and the other azathioprine. Both experimental groups either separately or combined showed significantly greater areas of colonic ischemic changes than did the control group. This study demonstrated that the systemic administration of immunosuppressive agents may augment the development of ischemic colitis [7].

The diagnosis of bowel ischemia is often difficult to

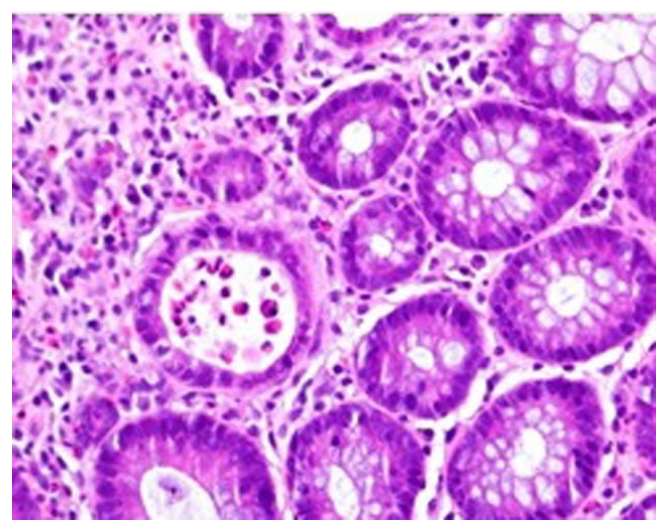

Figure 2. Dilated crypts filled with neutophils and necrotic debris.

make on the basis of plain radiography and barium studies. The common CT findings in mesenteric ischemia include dilated bowel, focal or diffuse bowel wall thickening, abnormal bowel wall enhancement (double halo or target sign), mesenteric edema, engorged mesenteric vessel and ascites. However, the lack of specificity of these signs is a limitation of CT because they can also be seen in patients with pancreatitis, mechanical bowel obstruction, peritonitis, or inflammatory bowel disease, all of which may mimic intestinal ischemia [8]. Therefore, in the clinical investigation of abdominal pain in SLE, involvement of multiple vascular territories on CT scans, in addition to improvement after intravenous prednisolone treatment and discontinuation of immunosuppressive agent, may favor a diagnosis of reversible ischemic bowel disease.

Managements of abdominal manifestations of SLE, in the absence of compelling radiographic or clinical findings suggestive of infarction or perforation, are steroid, antibiotics and fluid therapy [9].

The present case reveals ischemic change of edematous, erythematous mucosa and ulcerations in the cecum and small portion of the ascending colon with normal mucosa in the rest of the colon. Even though the patient has received immunosuppressive agents, she had stable disease activity during last three months without using steroid, with reported history of normal screening colonoscopy five years ago. Comparing with previous laboratory findings, several tests concerned with SLE disease activity demonstrated increased activity: elevated ESR, CRP and antinuclear antibody. Under this condition, we could diagnose ischemic colitis associated with increased SLE activity and vasculitis by histopathology. Generally, a typical pattern of vasculitis is quite difficult to prove by endoscopic biopsy [10]. In our case patient has clinical improvement with discontinuation of immunosuppressive therapy and conservative management without the initiation of steroids.

In conclusion, the present case demonstrated ischemic colitis associated with intestinal vasculitis and increased 
SLE activity, and the patient showed clinical improvement with conservative treatment and discontinuation of immunosuppressive treatment. In the presence of abdominal pain or bloody stool in patients with active SLE, ischemic colitis should be considered and treated accordingly.

\section{References}

1. Versaci A, Macri A, Scuderi G, Bartolone S, Familiari L, Lupattelli T, Famulari C. Ischemic colitis following colonoscopy in a systemic lupus erythematosus patient: report of a case. Dis Colon Rectum. 2005;48(4):866869.

2. Lafsky RD. Colonoscopy in ischemic enterocolitis. Gastrointest Endosc. 2000;52(2):310-311.

3. Miyahara S, Ito S, Soeda A, Chino Y, Hayashi T, Takahashi R, Goto D, et al. Two cases of systemic lupus erythematosus complicated with colonic ulcers. Intern Med. 2005;44(12):1298-1306.

4. Grimbacher B, Huber M, von Kempis J, Kalden P, Uhl M, Kohler G, Blum HE, et al. Successful treatment of gastrointestinal vasculitis due to systemic lupus ery- thematosus with intravenous pulse cyclophosphamide: a clinical case report and review of the literature. $\mathrm{Br} \mathrm{J}$ Rheumatol. 1998;37(9):1023-1028.

5. Lee CK, Ahn MS, Lee EY, Shin JH, Cho YS, Ha HK, Yoo B, et al. Acute abdominal pain in systemic lupus erythematosus: focus on lupus enteritis (gastrointestinal vasculitis). Ann Rheum Dis. 2002;61(6):547-550.

6. Cappell MS. Intestinal (mesenteric) vasculopathy. II. Ischemic colitis and chronic mesenteric ischemia. Gastroenterol Clin North Am. 1998;27(4):827-860, vi.

7. Gomella LG, Gehrken GA, Hagihara PF, Flanigan RC. Ischemic colitis and immunosuppression. An experimental model. Dis Colon Rectum. 1986;29(2):99-101.

8. Taourel PG, Deneuville M, Pradel JA, Regent D, Bruel JM. Acute mesenteric ischemia: diagnosis with contrastenhanced CT. Radiology. 1996;199(3):632-636.

9. Gore RM, Marn CS, Ujiki GT, Craig RM, Marquardt J. Ischemic colitis associated with systemic lupus erythematosus. Dis Colon Rectum. 1983;26(7):449-451.

10. Teramoto J, Takahashi Y, Katsuki S, Sato T, Sakamaki S, Kobayashi D, Watanabe N, et al. Systemic lupus erythematosus with a giant rectal ulcer and perforation. Intern Med. 1999;38(8):643-649. 